\title{
Material and Charge Transport of Large Organic Salt Clusters and Nanoparticles in Electrospray lon Beam Deposition
}

\author{
Gordon Rinke ${ }^{1}$, Ludger Harnau ${ }^{1}$, and Stephan Rauschenbach*1,2 \\ ${ }^{1}$ Department of Chemistry, University of Oxford, 12 Mansfield Road, Oxford OX1 3TA, UK \\ ${ }^{2}$ Max Planck Institute for Solid State Research, Heisenbergstr. 1, DE-70569 Stuttgart, Germany
}

Keywords: Soft Landing, Electrospray Ion Beam Deposition, Scanning Probe Microscopy, Cluster, Nanoparticle 


\begin{abstract}
Electrospray ion beam deposition (ES-IBD) or ion soft landing has been demonstrated as a technique suitable to process nonvolatile molecules in vacuum under perfectly controlled conditions, an approach also desirable for the deposition of nanoparticles. Here we present results from several approaches to generate, characterize, and deposit nanoparticle ion beams in vacuum for deposition. We focus on cluster ion beams generated by ESI of organic salt solutions. Small cluster ions of the salts appear in the mass spectra as defined peaks. In addition we find nanoparticle-sized aggregates, appearing as a low intensity background at high $m / z$-ratio, and show by IBD experiments that these clusters carry the major amount of material in the ion beam. This transition from clusters to nanoparticles and their successful deposition shows, that ES-IBD can in principle handle ion beams of very heavy and highly charged nanoparticles. In related experiments, however, we found the deposition of nanoparticles from dispersions to be of low reproducibility, due to the lack of control by mass spectrometry.
\end{abstract}

\title{
1 Introduction
}

Clusters or nanoparticles are objects, consisting of only a small number of atoms or molecules. They play an important role in nanotechnology due to the physical and chemical properties that arise from their high surface-to-volume ratio.[1] Composed of only few atoms or molecules, clusters have properties strongly dependent on the exact number of constituents, while the larger nanoparticles, is a nanometersized aggregate, where addition or removal of a few atoms or molecules neither alters its properties nor shape significantly. Recently advances in solution synthesis methods led to the fabrication of nanoparticles which can be highly complex in chemical composition, shape, and functionality. [2, 3]

While a large variety of clusters and nanoparticles is available, harnessing their properties for applications requires their integration in meso- and macroscopic systems such as surface coatings, thin films, or devices, which is mostly done by ambient processing from solution. Vacuum deposition of gas phase clusters and nanoparticles would offer a higher level of control and cleanliness, advantageous for device fabrication processes and optimising thin film properties for an application. Small atomic and molecular clusters, generated by condensation of vapor during its expansion into vacuum, [4] are one possible source of gas phase clusters widely used for the deposition clusters and nanoparticles to surfaces in vacuum as neutral cluster beam of a distribution of sizes or as size selected, ionised clusters. [5-9]

These conventional cluster and nanoparticle beam sources based on vapour condensation do not offer a general method for vacuum deposition, because they are neither compatible with nanoparticles in suspensions nor with all kinds of substances used for creation of nanoparticle or clusters. As an alternative, electrospray ionization (ESI) could serve as a gas phase particle source for clusters and nanoparticles. Currently, ESI is one of the most prominent ionisation methods for mass spectrometry (MS), i.e. method for generating gas phase ions, owning to its general applicability to almost any molecule ranging from small organic species (below $100 \mathrm{u}$ to the large biological species. For the generation of nanoparticle ions by ESI thus two distinct approaches can be envisioned: First, the generation of nanoparticle gas phase ions by electrospraying solutions or dispersions containing nanoparticles, and second, the creation of nanoparticles or clusters in the electrospray from a solution containing their constituents or precursors molecules.

Several examples demonstrated that, firstly, gas phase ions can be created from very large, nanometer sized particles objects of mega-Dalton masses present in solution while maintaining their integrity, and that, secondly, these heavy objects can be guided, handled and analysed by ion optics in vacuum. The ionisation and analysis of large protein assemblies[10] and entire viruses[11, 12] by native ESI, as well as the 
ionisation and trapping of nanoparticles for spectroscopy[13][14, 15] clearly show that ESI works for heavy, nanometre sized objects. Likewise, the generation of charged gas phase clusters from their constituents in solution is well documented for ESI.[16-19] Their components can be strongly interacting substances such as simple salts[20] or functional macromolecules like proteins.[21] Even big micellar structures, capable of encapsulating other large molecules, were evidenced by mass spectrometry, indicating that also large nanoparticles of complex composition may be created in the ESI process.[22]

Recent deposition experiments with organic and inorganic salt clusters in our laboratory showed the possibility to grow crystalline thin films and submonolayer coatings from cluster ion beams[23, 24], prompting to study efficiency of the growth process. Also small ligand capped gold clusters from a solution have been mass selected and soft landed in a very controlled manner.[25] The deposition of nanoparticles has been attempted using simple deposition sources, often with limited ion optics[26], showing that suspensions of carbon nanotubes[27] or CdSe particles[28] yield a surface coverage of individual particles, yet it is not certain wether gas phase ions or a jet of (charged) solvent droplets containing the particles (similar to pulsed valve inlets[29]) were deposited. This knowledge is hard to obtain for nanoparticle or large cluster beams, since the size and charge state distribution inhibits the identification of individual peaks in the mass spectrum.

In this work we explore the integration of large clusters and nanoparticles into vacuum processing via electrospray ion beam deposition (ES-IBD) with the aim of a controlled deposition. [30, 31] The applications for nanoparticle- or cluster- coated surfaces can be as diverse as, for instance, the fabrication of a sample for microscopic analysis[31-34] or the generation of a functional surface.[35-38] Depending on the application, for a deposition is it thus crucial to control coverage, beam composition, and surface interaction, while sufficient flux for the coating of a macroscopic surface must be supplied. This, foremost, requires access to the amount and distributions of material and charge among the particles of different sizes and charge state in the ion beam.

We use the organic salts Sodium Dodecyl Sulfate (SDS) and Sodium Citrate (SoCit) to form large clusters/nanoparticles in the electrospray and demonstrate their deposition by ES-IBD. With our ES-IBD setup we are able to determine the ratio of material and charge in the particle beam by two independent approaches: first, in-situ time-of-flight mass spectrometry (TOF-MS) capable of detecting the distribution of cluster ions, and second, a combination of ES-IBD and atomic force microscopy (AFM) gives quantitative access to the net deposited charge and the net amount and spatial distribution of deposited material. The first method requires an in depth analysis of the mass spectra, for the second method the morphology of the structures created by the ion beam deposition are analyzed in order to quantify the material amount. Moreover, SDS and SoCit are known to encapsulate and stabilize nanoparticles: SDS, for instance, is used to disperse carbon nanotubes (CNT) and SoCit to stabilize gold nanoparticles in solution. Based on the experience gained on the deposition of large clusters, we can comment on the the controlled deposition of nanoparticles from suspensions and dispersions.

We find that organic salts generate clusters ranging from the smallest possible charged aggregate to nanoparticles of hundreds of molecules. Both, mass spectrometry and deposition experiments show that the small, low charge state ions contribute the main fraction of the charge in the beam, while heavy, high charge state ions contribute the major amount of material. As a consequence we were able to perform well controlled depositions at high flux and achieved thin films and nanostructured island growth at the surface. Moreover, this implies the general possibility of fabricating nanoparticle coatings by ES-IBD at a similar level of control as for molecular ions, provided that stable particles are created in the electrospray or 
ionized from a solution, dispersion, or suspension. Unfortunately, depositions of nanoparticles stabilised in suspension by detergents like SDS or SoCit as well as the deposition of nanoparticles from detergent-free solutions was not successful or reproducible, respectively. However, the success of detergent optimisation in native mass spectrometry[39, 40] and the recent advances in single particle $M S[14,41]$ will allow to better understand and optimise the ion generation of these systems, expanding the scope of ES-IBD to nanoparticle deposition.

\section{Results and Discussion}

\subsection{Organic Salt Cluster Formation}

Cluster ion beams of positively charged SDS and negatively charged SoCit are generated by pneumatically assisted ESI from solution and are analyzed by time-of-flight mass spectrometry (TOF-MS) to identify the clusters and study the fragmentation behavior. Typical mass spectra are shown in Fig. 1 (a) and (b).

a) Sodium Dodecyl Sulfate (SDS)
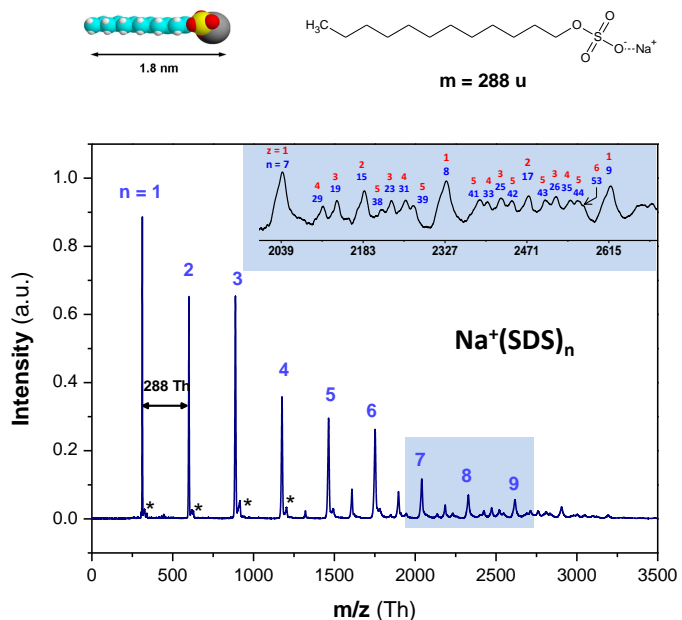

c) SDS concentration dependence

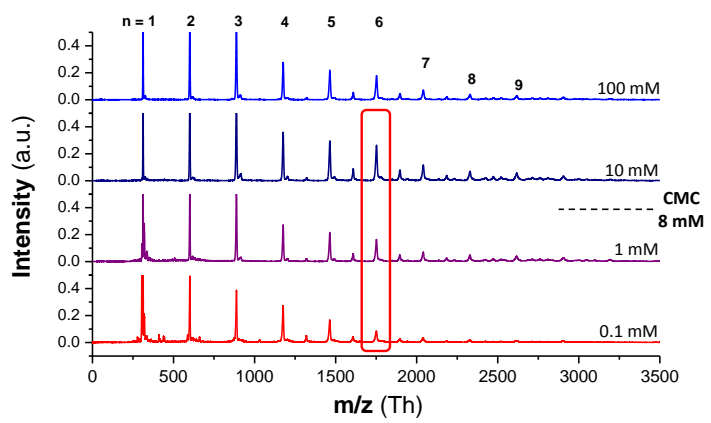

b) Sodium Citrate (SoCit)
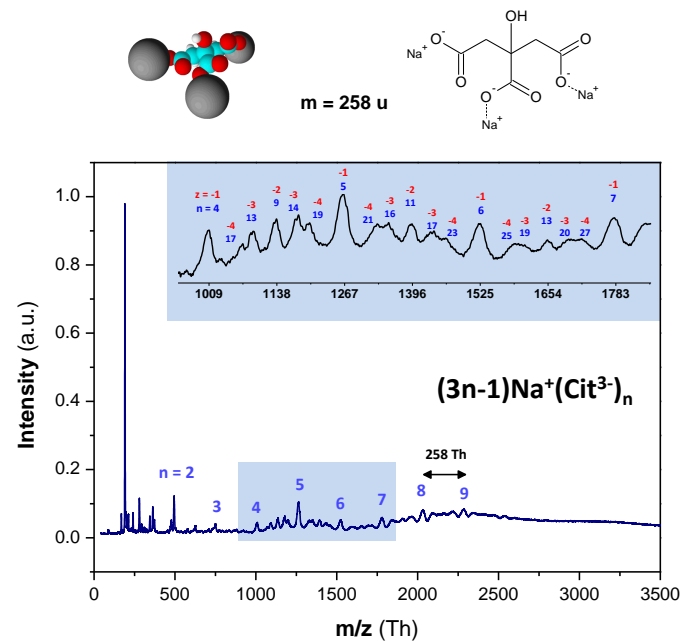

d) SoCit concentration dependence

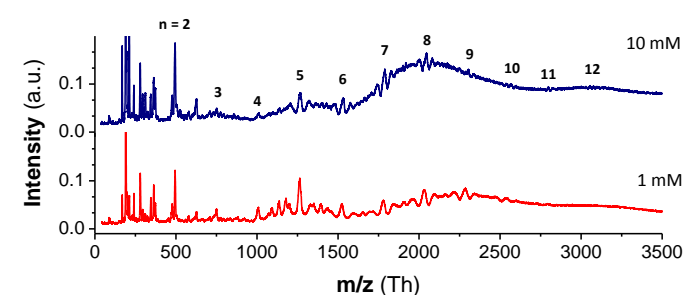

Figure 1: Mass spectra and concentration dependence of the SDS and SoCit cluster beams. $(a, b)$ ESI-TOF mass spectra of SDS (a) and SoCit (b). Peaks for singly charged clusters are most pronounced. Inset: Magnification of high $\mathrm{m} / \mathrm{z}$ area showing multiply charged clusters. (c,d) Mass spectra of SDS (c) and SoCit (d) at different concentration. The relative intensity of larger cluster peaks increases.

The mass spectra of SDS (Fig. 1a) show several series of peaks, which can be identified as SDS cluster ions. In the most pronounced series with the largest periodicity, peaks are separated by $288 \mathrm{Th}^{\mathrm{i}}$, the mass of the SDS molecule. These peaks thus correspond to singly charged clusters of the type $\mathrm{Na}^{+}(\mathrm{SDS})_{\mathrm{n}}$,

\footnotetext{
'We use Thomson as unit of mass-to-charge-ratio where $1 \mathrm{Th}=1 \mathrm{u} / \mathrm{e}[42]$
} 
where $n$ is the number of SDS molecules. Inbetween the single charged cluster peaks, additional series of lower periodicity can be observed. They are characteristic for multiply charged clusters $(z>1)$ and thus separated by fractions of 288 Th, i.e. 144 Th for $z=2,96$ Th for $z=3$ etc. The largest identified cluster consists of 53 molecules with a charge state $z=6$. Furthermore, a small contamination of potassium is detected in the SDS solution, visible as peaks shifted by 16 Th (some are indicated by "*" in Fig. 1a), which is the $m / z$ difference of one $\mathrm{Na}^{+}$ion replaced by one $\mathrm{K}^{+}$ion in the single charged SDS cluster. Overall, the high purity of the cluster ion beam especially by the absence of solvent is verified, which is important for its application in deposition.

Similar observations can be made in the mass spectra of SoCit in Fig. 1(b) where the majority of peaks corresponds to cluster ions as well. The main periodic peak series is separated by $258 \mathrm{Th}$, relating to a single neutral SoCit molecule $\left(3 \mathrm{Na}^{+} \mathrm{Cit}^{3-}\right)$. The peaks in the range $190-450 \mathrm{Th}$ correspond to the singly charged citrate molecules and to contaminants. Like in the case of SDS, peaks of higher charged, larger clusters appear inbetween the singly charged cluster peaks.

One parameter which can affect the cluster formation is the concentration of the solution.[18] The influence of the concentration on the cluster size is studied by varying the solution concentration of SDS (0.1 $100 \mathrm{mM})$ and of SoCit $(1-10 \mathrm{mM})$ for a constant declustering potential $\mathrm{V}_{\text {decl }}$ of $175 \mathrm{~V}$ in the nozzleskimmer region of the instrument, a value for which fragmentation is relatively negligible, only noticeable when comparing logarithmic plotted mass spectra .

Increasing the concentration results in a higher relative intensity of peaks in the mass spectra of both substances at higher $m / z$ values, while the spectra remain similar (Fig. 1 (c) and (d)). In the case of the amphiphilic SDS the increase of the concentration above the critical micelle concentration (CMC) of $c_{c m c}=8.3 \mathrm{mM}$,[43] where micelles have formed in solution, has only a minor effect on the appearance of the mass spectra. Small changes are observable for instance in the intensity of low molecular weight clusters like $n=6, z=1$, which increases with concentration for $c \leq c_{c m c}$ (see Fig. 1(c) red box). In this concentration regime $\left(c \leq c_{c m c}\right)$, clustering of SDS solute molecules is enhanced with increasing concentration due a decreasing mean intermolecular distance in the homogeneous solution. Above $c_{c m c}$ the contribution of the low molecular weight clusters decreases and a very broad unresolved region at $m / z>3500$ Th consistent with large aggregates can be found (see Supp. Info., Fig. S1).

In water, the molecules form micelles above the critical micelle concentration and can form aggregates with approximately 50 SDS molecules $(14400 \mathrm{u}$ ).[44] The SDS molecules are aligned with their charged head groups facing towards the water, creating a water free environment inside the micelle. Transferred into the gas phase these regular micelles are expected to rapidly invert into reverse micelles. This happens because of the water evaporation during the ESI process, shown in computer simulation studies on cetyltrimethylammonium bromide micelles[45] and dodecyl phosphocholine micelles. [46] In reverse micelles the charged head groups are oriented towards the core, while the alkyl tails point outwards. Alternatively, reverse micelles may be transferred into the gas phase as a whole encapsulating water.[22] However, the mass spectra in Fig. 1 show only peaks for SDS without any encapsulated water so that the reverse micelle configuration can be assumed for our SDS cluster ion beams.

Furthermore, it is observed that the intensity of the singly charged SDS cluster peaks below $3500 \mathrm{Th}$ decreases exponentially as the cluster size increases. This behavior is indicated by a straight line in the logarithmic plot of the mass spectra in Fig. 2(a). This feature shows that the cluster formation follows a so-called most probable size distribution mechanism.[47] In this case, the energy associated with the 
attachment of one SDS molecule to an agglomerate, does not depend on its size, which is in contrast to magic number clusters.[48] The reason for absence of magic number clusters is likely the large size and flexibility of the molecule and hence in the large amount of configurations of the organic salt cluster or which none are especially pronounced in energy.

The structure of SoCit does not promote the formation of micelles in solution or in vacuum due to its non-amphiphilic nature. The exact shape of the clusters is therefore expected to be that of a small crystallite, strongly disturbed by excess charge and by the large surface-to-volume ratio. Similar to SDS, increasing the concentration of the SoCit solutions causes a slight shift of the relative intensity towards higher $m / z$ values, indicative of increasing cluster size.

\subsection{Fragmentation of Organic Salt Clusters}

Fragmentation of molecules and aggregates is an inherent feature of mass spectrometry and just as effective on the final content of the cluster beam as the parameters of the ion formation like concentration or the contents of the solution. In our setup fragmentation is achieved by inducing collisions with the background gas in the first pumping stage at $0.1 \mathrm{mbar}$ by adjusting the declustering potential $V_{\text {decl }}$, between nozzle and skimmer or nozzle and ion funnel. Upon increasing this potential, collisions become more intense and lead to fragmentation of the ionized clusters into smaller clusters, while the covalent bonds of the molecules constituting the cluster are not affected.

The influence of the declustering potential on SDS and SoCit clusters is shown in Fig. 2(a) for $10 \mathrm{mM}$ SDS and in Fig. 2(b) for $1 \mathrm{mM}$ SoCit solutions. The mass spectra are plotted on a logarithmic intensity scale in order to emphasize the intensity above $3500 \mathrm{Th}$, which would not be visible if the spectra were scaled linearly and display the full intensity range.

For both SDS and SoCit the mass spectra show the most intense peaks below $3500 \mathrm{Th}$ or $1500 \mathrm{Th}$, respectively. Many closely spaced peaks corresponding to high-charge-state clusters are observed in the SDS spectra for $m / z$ values ranging from $1500-3500 \mathrm{Th}$. In the SoCit spectra mainly single charged peaks are seen for low declustering potentials. Additionally a broad feature of low intensity without resolved peaks can be seen in the high $\mathrm{m} / z$ - range between $3500 \mathrm{Th}$ and $7000 \mathrm{Th}$ for SDS and beyond $1500 \mathrm{Th}$ for SoCit (marked with "*" in Fig. 2).

Upon increasing the declustering potential, the broad feature shifts towards higher $\mathrm{m} / \mathrm{z}$-values and eventually the series of singly charged peaks is seen in the mass spectra of both SDS and SoCit. In addition, at high $m / z$ values, doubly and triply charged SDS clusters of low intensity can be distinguished (indicated by arrows in Fig. 2(a)).

Hence, the evolution of the mass spectra as a function of the declustering voltage can be explained by the disintegration of big clusters $(n>50)$ of high charge state $(z>6)$. Due to the limited resolution of the instrument, their dense distribution of peaks at low declustering potential cannot be resolved. Upon increasing the potential, these clusters are fragmented into smaller clusters of lower charge state, which now appear in the mass spectra at higher or lower $m / z$ values around the original cluster peak. Even though large clusters can tolerate more energy imparted from collisions and by Coulomb repulsion due to a larger number of degrees of freedom and larger size, respectively, the relative abundance of the high charge state clusters is found to be reduced in the experiments. For them fission can be more probable because highly charged clusters gain more energy in the electric field between subsequent collision events and are additionally destabilised by Coulomb repulsion. Since smaller, less charged clusters are the outcome of all fission events, ultimately the material in the cluster beam becomes distributed mostly into singly charged 
(a) SDS

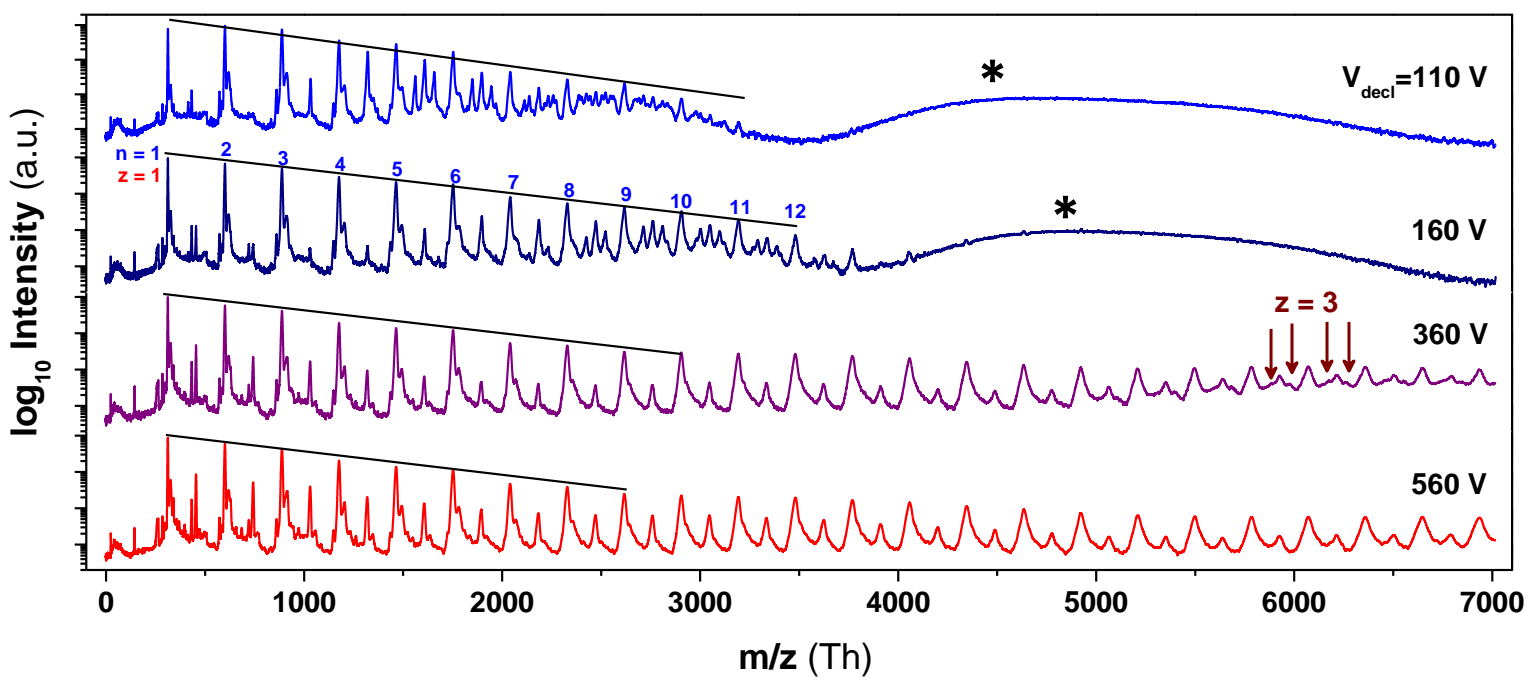

(b) SoCit

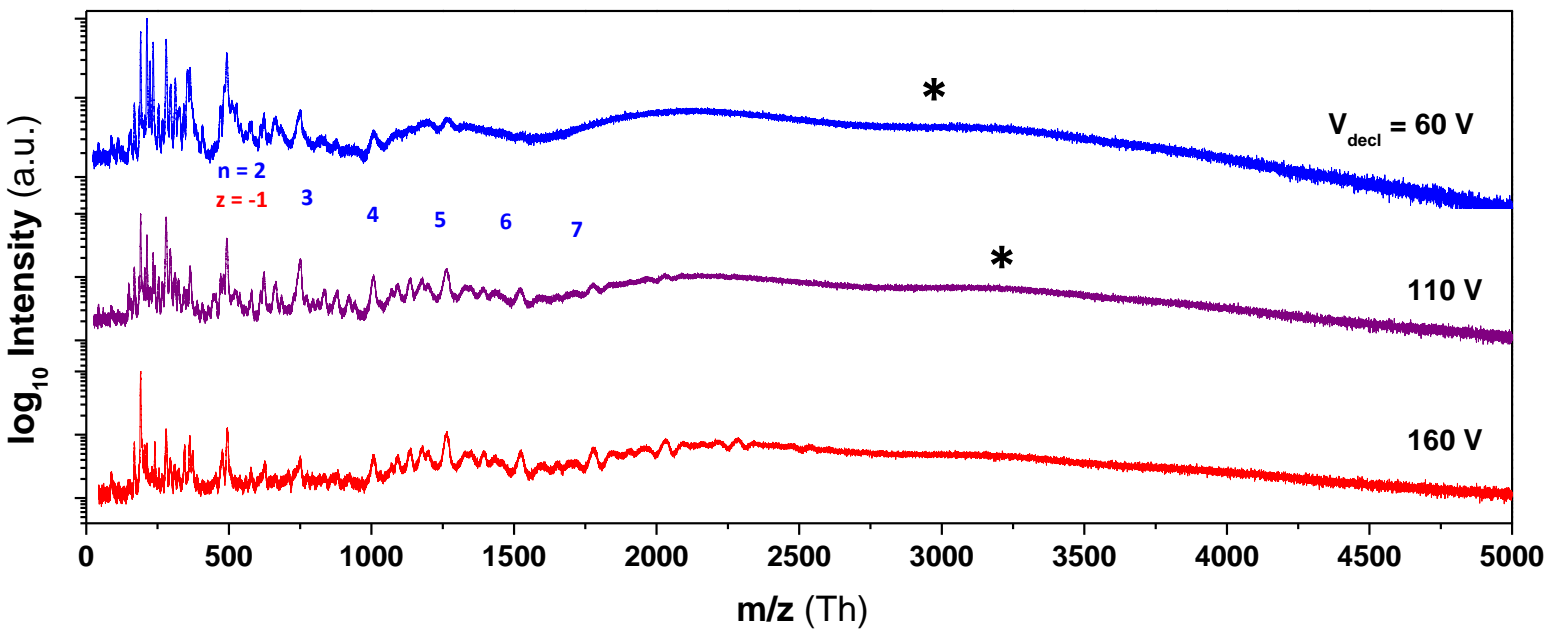

Figure 2: Fragmentation mass spectra of SDS and SoCit: (a) Mass spectra of positively charged SDS from a $10 \mathrm{mM}$ solution at several declustering potentials $V_{\text {decl }}$ (intensity in log-scale). Red arrows in the mass spectrum for $V_{\mathrm{decl}}=360 \mathrm{~V}$ indicate some of the triply charged clusters at high $\mathrm{m} / z$. The straight lines highlight the exponentially decreasing abundance of the singly charged clusters. (b) Mass spectra of negativly charged SoCit from $1 \mathrm{mM}$ solution at several declustering potentials $V_{\mathrm{decl}}$. The $(*)$ in both spectra marks regions of unresolved intensity of highly charged clusters.

clusters as the declustering potential increases (see Fig. 2(a), $V_{\text {decl }}=560 \mathrm{~V}$ ).

The clusters at the center of the unresolved intensity in the SDS spectra at approx. 5000 Th correspond to a single charged cluster with 17 molecules, or could be attributed to clusters with roughly 85 molecules, if the highest observed charge state $z=6$ is assumed. The integrated intensity of the mass spectraii shows that this region accounts for $j_{h i}=9 \%$ of the detected particles, i.e. counts detected in the MS (see Supp. Info., Fig. S3). However, due to their size and depending on their charge state, the amount of material of this region can be significant and is not intuitively deduced from the mass spectra.

\footnotetext{
ii Integrated counts, normalized to the bin width in $\mathrm{m} / \mathrm{z}$, because in TOF bin width is constant in time-of-flight.
} 


\subsection{Material and Charge Distribution in the Mass Spectra}

Independent of charge state and size, each cluster is registered in the mass spectrometer as one count. To obtain quantitative information about the material-to-charge ratio of a cluster ion beam from mass spectrometry, the material and charge contribution of each individual charge peak must be considered separately. Because the peak series for the various charge states are partially overlapping in the mass spectra when $\mathrm{m} / \mathrm{z}$ is the same, we fit the experimental data with a model function containing all charge states and cluster sizes. As a result the intensity of each cluster ion species, defined by number of molecules and charge state, is known and the material and charge contribution to the beam can be calculated. The unresolved region of the mass spectrum has to be excluded from the fitting procedure, due to the fact that here well-defined peaks are not present and thus the result would be arbitrary. Nevertheless, based on the detected intensity the results of the fitting of the resolved part can be extrapolated to assign charge states to the clusters in this region and hence estimate the material content.

The mass spectrum of SDS is fitted in the range from 0 Th to 3500 Th with the intensity function $J(M)$, (Eqn. 1) where $M \equiv m / z$. This function generates mass spectra of SDS clusters of different charge states, size and intensity, by adding up all possible clusters as Gaussian peaksiii $g\left(M, \frac{m_{n, z}}{z}, \frac{m_{n, z}}{z R}\right)$ characterized by number of molecules $n$ and charge state $z$ with mass $m_{n, z}=z m_{\mathrm{Na}}+n m_{\mathrm{SDS}}$. An envelope Gaussian distribution $J_{z} g\left(n, c_{z}, w_{z}\right)$ is multiplied, serving as simple approximation for the intensity distribution of each charge state series.

$$
J(M)=\sum_{z=1}^{z_{\max }} \sum_{n=1}^{n_{\max }} J_{z} \cdot g\left(n, c_{z}, w_{z}\right) \cdot g\left(M, \frac{m_{n, z}}{z}, \frac{m_{n, z}}{R z}\right)
$$

The parameters intensity, $J_{z}$, the center position, $c_{z}$, and the width, $w_{z}$, are fitted for each charge state series $z=1 \ldots z_{\max }$. The difference in peak width is introduced in the model through a parameter $R \equiv m / \Delta m$, in which instrumental resolution and isotopic broadening are merged into one number that is fitted as well. To consider the low abundance peaks, the fit is performed using $\log J(M)$ as fitting function on logarithmically scaled data.

Figure 3(a) shows the measured mass spectrum of SDS in good agreement with the fitted spectrum for charge states $z=1 \ldots 5$. In addition, reasonable values for resolution demonstrates the feasibility of the fitting procedure. Below the mass spectra of each charge state series, extracted from the fit, are displayed individually. Summed up they yield the fitted spectrum; separated they allow to access the contribution of the different charge states to each peak. It becomes obvious that many of the heavier cluster peaks are in fact mostly composed of multiple charge state clusters, because the intensity of the $z=1$ series peaks becomes very low beyond $2100 \mathrm{Th}$. The deviation between fit and data for high $\mathrm{m} / \mathrm{z}$ is owned to the relatively low abundance of high charge state peaks as well as to the simplicity of the model, in which the Gaussian envelopes are merely a simple approximation. Also, the resolution of the mass spectra does not merit a systematic study because the most interesting high $\mathrm{m} / \mathrm{z}$ region is not sufficiently resolved, here we only discuss one typical SDS mass spectrum which nevertheless illustrates how material and charge are distributed.

The result of the fit is summarized in Fig. 3(b) (see supporting infomation S4.2 for more details). The relation between $\mathrm{m} / \mathrm{z}$-ratio and charge state of the clusters is shown in the left panel, where the center position the envelope $c_{z}$ and width $w_{z}$ is plotted. The $\mathrm{m} / \mathrm{z}$ ratio of the clusters rises with increasing charge

\footnotetext{
iii $g\left(x, x_{0}, \sigma\right)=\frac{1}{(\sigma \sqrt{2 \pi})} \exp \left[-\frac{1}{2}\left(\frac{x-x_{0}}{\sigma}\right)^{2}\right]$
} 
a)

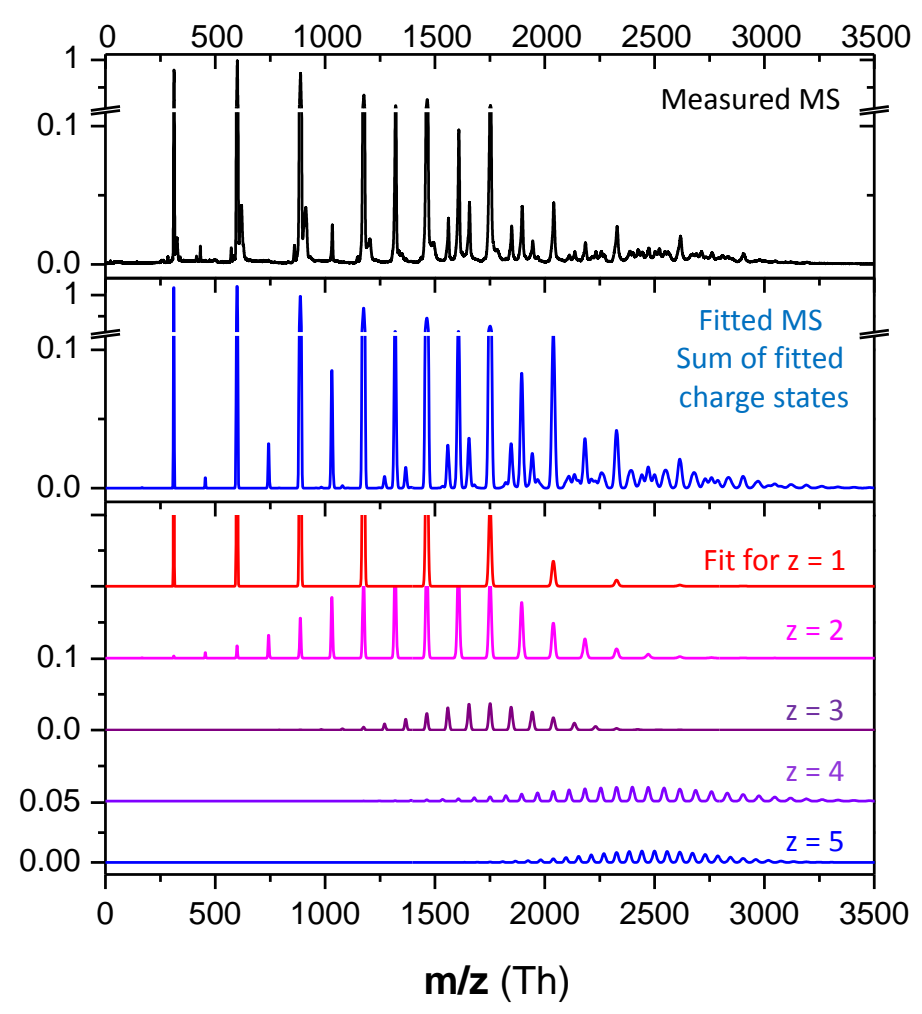

b)

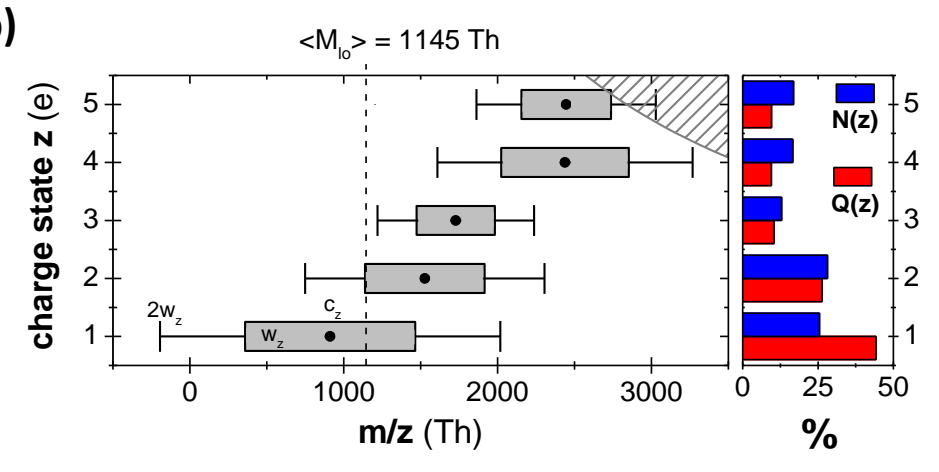

Figure 3: Material $N(z)$ and charge content $Q(z)$ for each cluster charge state distribution from fitting of the mass spectral intensity of SDS:(a) SDS mass spectrum $\left(10 \mathrm{mM}, V_{\mathrm{decl}}=110 \mathrm{~V}\right)$ and best fit including charge states up to $z=5$ (blue). Below, contributions of the charge states up to $z=5$ shown individually. (b) $\mathrm{m} / \mathrm{z}$-width and center position of each charge state up to $z=5$ as found from the fitting. The shaded area indicates the resolution limit of the mass spectrometer. The right panel show the relative material content $N(z)$ and the relative charge amount $Q(z)$ as a function of charge state.

state $z$, while the width of the distribution stays roughly constant. The shaded area in the top right corner of that plot represents the resolution limit of our mass spectrometer $(R=300)$, illustrating that charge states larger than $z=5$ can not be resolved beyond 3000 Th.

Once we know size and charge state of the clusters, the intensity parameter $J_{z}$ can be used to calculate more intuitive measures for the intensity (see supporting information S4.2). In the right panel of Fig. 3(b) the relative amount of material $N(z)$ and charge $Q(z)$ are shown as function of charge state $z$. Stunningly, the singly charged clusters, which visually dominate the mass spectrum at linear scale, contain only $25 \%$ of the beams material flux, compared to $45 \%$ of the charge. In contrast, each of the higher charge states $z=3-5$ contributes only $\approx 10 \%$ of the charge but almost $20 \%$ of the material. Weighted by the number 
of particles, the fitted mass spectrum up to $3500 \mathrm{Th}$ with the resolvable charge states up to $z=5$, has an average charge state of $\bar{z}_{l o}=1.54$ and an average $m / z$-ratio of $\left\langle M_{l o}\right\rangle=1145 \mathrm{Th}$, which corresponds to 4.0 molecules per charge in the cluster beam.

In summary, high charge states that are almost invisible in the mass spectrum contribute significantly less charge but a relatively large amount of material. This suggests that the unresolved region (3500 $7000 \mathrm{Th}$ ), which represents only $j_{h i}=9 \%$ of the mass spectrometric intensity, contains a significant amount of material but rather little charge, even if many ions have charge states higher than $z=5$. This would strongly affect the average $m / z$-ratio of the overall cluster ion beam, $\left\langle M_{t o t}\right\rangle$. We estimate this value as the weighed average $m / z$-ratio of the resolved part at low $\mathrm{m} / \mathrm{z}$-ratio, $\left\langle M_{l o}\right\rangle$, and of the unresolved part at high $\mathrm{m} / \mathrm{z}$-ratio of the mass spectrum, $\left\langle M_{h i}\right\rangle$.

$$
\left\langle M_{t o t}\right\rangle\left(\bar{z}_{h i}\right)=\frac{\left\langle M_{l o}\right\rangle \cdot j_{l o} \cdot \bar{z}_{l o}+\left\langle M_{h i}\right\rangle \cdot j_{h i} \cdot \bar{z}_{h i}}{j_{l o} \cdot \bar{z}_{l o}+j_{h i} \cdot \bar{z}_{h i}}
$$

The evaluation of $\left\langle M_{t o t}\right\rangle$ further requires knowing the average charge states in the lwo and high regions, $\bar{z}_{l o}, \bar{z}_{h i}$, and the relative intensities, $j_{l o}$ and $j_{h i}$, all of which can be extracted from the mass spectrum except for $\bar{z}_{h i}$ :

In Fig. 4 (a) the overall average $\mathrm{m} / \mathrm{z}$-ratio of the ion beam $\left\langle M_{\text {tot }}\right\rangle$ is plotted (red line) as a function of $\bar{z}_{h i}$ for $\left\langle M_{l o}\right\rangle=1145 \mathrm{Th}$ and $\bar{z}_{l o}=1.56$, taken from the fit of the resolved region, and $\left\langle M_{h i}\right\rangle=5000 \mathrm{Th}$ and $j_{h i}=9 \%$ measured from the mass spectrum (see supporting info S4.3 and Fig. S3). For this plot Eqn. 2 was evaluated for the average charge states of the unresolved clusters $\bar{z}_{h i}=1 \ldots 50$, a range chosen based on charge states known for large objects of comparable size such as proteins.

With increasing charge state $\bar{z}_{h i}$ the contribution of the unresolved part of the mass spectrum of $9 \%$ of the counts becomes more relevant and $\left\langle M_{t o t}\right\rangle$ shifts significantly towards $\left\langle M_{h i}\right\rangle$. For instance for assuming $\bar{z}_{h i}=1$ yields an average $m / z$ ratio of $\left\langle M_{t o t}\right\rangle=1375 \mathrm{Th}$, it has already doubled for $\bar{z}_{h i}=10$. Even if only background (chemical noise) was detected in the high $\mathrm{m} / \mathrm{z}$-region, for which we assume an intensity ratio of $1 \%$, the average $m / z$-ratio $\left\langle M_{\text {tot }}\right\rangle$ doubles for high charge states.

The impact of the unresolved intensity of $j_{h i}=9 \%$ on the material transport is illustrated in Fig. 4 (b) showing the material fraction of the high $\mathrm{m} / \mathrm{z}$-range as a function of the assumed charge state for the high $m / z$-range (solid line). At low charge states $\bar{z}_{h i}$, the major share of material is carried by low $m / z$ clusters. More likely, however, are moderate or high charge states for the heavy clusters. Already for $\bar{z}_{h i}=3.7$, the average value found for fitting the resolvable peaks, the material transport is equally divided between the low and the high $m / z$-range clusters. From here on the material transport fraction of the high $m / z$-range clusters increases to over $90 \%$ for $\bar{z}_{h i}=50$. Supposing again an intensity fraction of $j_{h i}=1 \%$ (dashed line), which would represent the (chemical) noise, the increase is less pronounced, but still the point of equal material fraction is found for a reasonable number of charges per cluster $\bar{z}_{h i} \approx 35$.

This analysis underpins the importance of the high $m / z$-region on the material transport in the ion beam. Providing that highly charged clusters are present in the ion beam, the material contribution of the unresolved region at high $\mathrm{m} / z$-ranges can easily dominate, even at low relative intensities in mass spectrometry. The results from the fit show that we have to expect high charge states the high $\mathrm{m} / \mathrm{z}$-region $\left(\bar{z}_{h i}>5\right)$, for which our estimate predicts an average $m / z$-ratio above 2000 Th and a material fraction of the high $m / z$-range of more than $60 \%$. This discrepancy is nicely illustrated by considering the fact that a SDS cluster of charge state $z=10$ at 5000 Th, containing approx. 85 SDS molecules, is detected as a single count in the MS, just as the single charged single molecule SDS $-\mathrm{Na}^{+}$ion. 


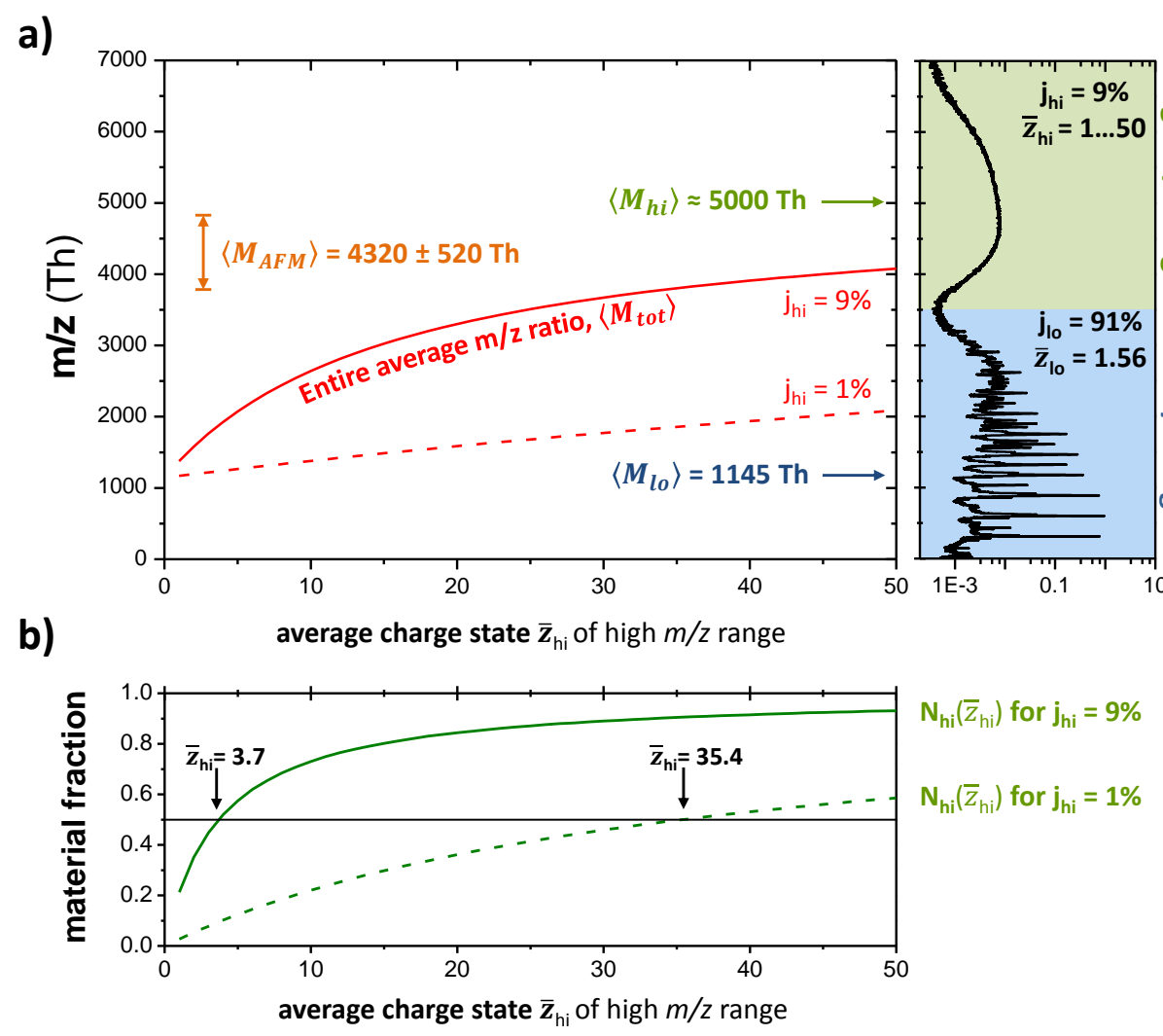

Figure 4: Estimation of charge and mass in unresolved MS region. (a) Average $m / z$ ratio $\left\langle M_{\text {tot }}\right\rangle$ as a function of the average charge state $\bar{z}_{h i}$ in the high $\mathrm{m} / \mathrm{z}$-range for an intensity fraction of $j_{h i}=9 \%$ as found from the mass spectrum (solid line). For comparison $j_{h i}=1 \%$ is assumed (broken line), which would represent the integrated noise level of the MS. (b) The material fraction of the high $\mathrm{m} / \mathrm{z}$ range in dependence of $\bar{z}_{h i}$ for $j_{h i}=9 \%$ and $1 \%$.

This analysis, done for a typical SDS data set, can be assumed to represent the material transport properties for similar cluster beams like the one of SoCit. The actual charge state distribution can, however, not be found with this method. Nevertheless, we see that low intensity clusters in the high- $m / z$ range can contribute the major amount of material and thus have to be considered in cluster ion beam deposition processes as the dominating component. On the one hand, this can be utilized in order to enhance the material flux and to prepare high coverage samples. On the other hand, contaminations barely observed in mass spectrometry should be avoided by mass selection. While they may not be observed in the mass spectrum they can nonetheless contribute a significant amount of material from large highly charged clusters.

\subsection{Statistical Analysis of the Deposited Material Amount}

To test the assertion that the high $m / z$-region, which is almost not visible in the mass spectra, dominates the material transport, the average $m / z$-ratio $\left\langle M_{t o t}\right\rangle$ can be measured through ion beam deposition experiments. The material amount is measured as the volume of the deposited material by AFM and the current integrated during deposition yields the net charge. This approach is entirely independent from mass spectrometry and additionally yields information about the structure and morphology of the deposited material from large cluster/nanoparticle beams at the surface. Thus it is the method of choice to validate our findings and to determine the average charge state of the high $\mathrm{m} / \mathrm{z}$-range $\bar{z}_{h i}$. 

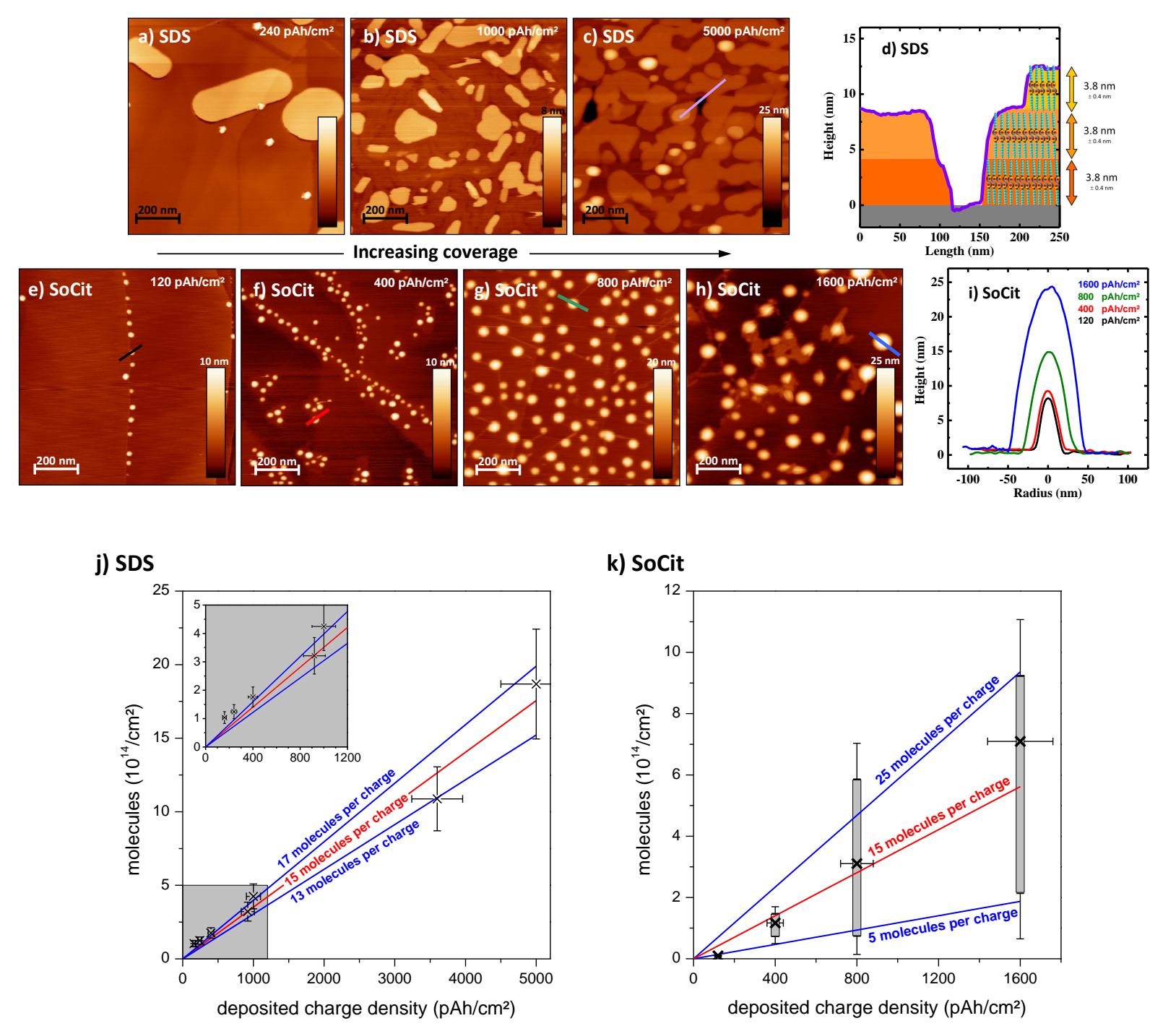

Figure 5: AFM images and coverage analysis of SDS and SoCit deposited as cluster beams on HOPG. AFM images with characteristic height profiles of SDS $(a-d)$ and SoCit $(e-i)$ on HOPG for increasing coverage, e.g. deposited charge. SDS grows as inverse double layers. SoCit forms 3dimensional islands. Molecular coverage, calculated from AFM images given as area density, plotted against the deposited charge for SDS (j) and SoCit ( $k$ ). Linear regressions yield average charge states of 15 molecules per charge for both SDS and SoCit.

Several samples of different coverage were prepared for both molecular cluster/nanoparticle beams by ES-IBD on highly oriented pyrolytic graphite (HOPG) substrates and characterized by AFM operated in tapping mode at several spots in ambient conditions. For all samples the delcustering potential was set to $\mathrm{V}_{\text {decl }}=110 \mathrm{~V}$ for SDS and $\mathrm{V}_{\text {decl }}=60 \mathrm{~V}$ for SoCit and the clusters were soft landed with $5 \mathrm{eV}$ per charge. The deposited charge density was ranging from $120 \mathrm{pAh} / \mathrm{cm}^{2}$ to $5000 \mathrm{pAh} / \mathrm{cm}^{2}$. The rf-quadrupole ion guides are operated in rf-only mode so that there is a lower $m / z$ cut off but no upper transmission limit. In the case of SDS the lower $m / z$ cut off is below the first SDS cluster at 311 Th while for the SoCit the cut-off is set at $500 \mathrm{Th}$ excluding the singly charged SoCit variations and possible contaminations.

On the surface the original SDS clusters are mobile and merge into larger flat aggregates, which upon increasing the coverage are stacked on top of each other (Fig. $5 \mathrm{a}-\mathrm{d}$ ). AFM images show that these islands have a height of $3.8 \mathrm{~nm}$ each, which is attributed to a double layer formation of the SDS molecule having a length of $1.9 \mathrm{~nm}$.[49] Due to the missing hydrophilic-hydrophobic interaction in vacuum, the 
ionic-polar interaction dominates the growth so that the molecules are arranged in an inverse membrane configuration. Further analysis with $\mathrm{X}$-ray diffraction and scanning tunneling microscopy show that these layers are crystalline.[23]

In contrast, SoCit forms three dimensional aggregates (Fig. 5 e-i). At low coverage small round islands are found at the step edges of the graphite (Fig. 5 e). A further increase of the coverage, leads to more islands distributed around the step edges and patches of several islands on the terraces (Fig. $5 \mathrm{f}$ ) until finally the islands are distributed homogeneously across the surface (Fig. $5 \mathrm{~g}$ ). Upon doubling the coverage very large and inhomogeneously distributed islands are observed where some of them are connected by flat rods (Fig. $5 \mathrm{~h}$ ). This growth sequence shows clearly that at higher coverage islands grow at the expense of smaller ones. The flat rods at higher coverage might indicate the formation of a second crystalline phase.

In both cases upon landing the gas phase clusters dissolve and rearrange on the surface. This mobility implies the possibility to use cluster ion beams for high flux crystalline growth from molecular ion beams, as shown for SDS[23], but unfortunately hinders us to determine the size of the individual cluster after deposition. Nevertheless, the number deposited molecules can be estimated, and, in combination with the deposited charge, an average cluster size in molecules per charge can be deduced.

The quantity of deposited SDS molecules is approximated by measuring the area coverage of the double layer and relating it to the molecular footprint. This is done by using the anhydrous SDS crystal structure, with an average area per polar head group of $19.3 \AA^{2}$ and a lamellar thickness of $1.9 \mathrm{~nm}$. [49] In the case of the SoCit the volumes of the islands are evaluated. For the approximation of the amount of SoCit molecules a density of $1.76 \cdot 10^{-6} \mathrm{~g} / \mathrm{m}^{3}$ is used. For the calculation a homogeneous coverage across the whole sample with an area of $12.5 \mathrm{~mm}^{2}$ can be assumed, which ensured by a wide deposition spot and is confirmed by the AFM measurements at different spots of the sample.

The analysis of many experiments yields a relation between the amount of deposited molecules and the deposited charge, depicted in Fig. 5 (a) and (b). For both molecules, the material amount increases with the deposited charge. Assuming a linear relationship, the slope of the curves corresponds to the average amount of molecules per charge, which is $15 \pm 2$ for SDS and $15 \pm 10$ for SoCit equating to an average $\mathrm{m} / z$ ratio of about $\left\langle M_{\mathrm{AFM}}^{\mathrm{SDS}}\right\rangle=4320 \pm 580 \mathrm{Th}$ and $\left\langle M_{\mathrm{AFM}}^{\mathrm{SoCit}}\right\rangle=3850 \pm 2500 \mathrm{Th}$, respectively. A large variance of the data is seen, especially for the SoCit data. The errors can be explained by the inherently inaccurate measurement of the island volumes from the AFM images caused by finding an appropriate base plane. By comparison, the determination of the amount of deposited SDS molecules proceeds through an area measurement, which is more precise.

Comparing the estimate for the average $m / z$ ratio $\left\langle M_{\text {tot }}\right\rangle$ to the measured $\left\langle M_{\mathrm{AFM}}^{\mathrm{SDS}}\right\rangle$ of about $(4320 \pm$ $580) \mathrm{Th}$, it is seen that for values of $\bar{z}_{h i}=30 \ldots 50,\left\langle M_{t o t}\right\rangle$ is located in the error margin of $\left\langle M_{\mathrm{AFM}}^{\mathrm{SDS}}\right\rangle$ (see Fig. 4a). Thus, it can be concluded that clusters with 30,50 or even more charges are present in the upper $\mathrm{m} / \mathrm{z}$ range of the mass spectrum. Conversely this means, that at this charge state and for an average cluster size of 15 molecules per charge, several hundred molecules compose one cluster, which then is an object with a diameter in the nanometer range and indeed can be considered as a nanoparticle. Comparing these data, one has to consider that the mass spectra represent a single measurement of an ion beam, while $\left\langle M_{\mathrm{AFM}}^{\mathrm{SDS}}\right\rangle$ is an average value over several experiments, hence several beams each prepared and optimised individually. Furthermore, the mass spectra might be incomplete at high $\mathrm{m} / \mathrm{z}$ ratios due to the instrument's limitation, so that the reach of the high $m / z$-range might be underestimated and even larger $\left\langle M_{h i}\right\rangle$ could be assumed. 


\section{Ionization and Deposition of Nanoparticles from Dispersions}

The work with the SDS and SoCit clusters showed, that in principle large nanoparticles can be transmitted to the sample surface in high vacuum. To extend on this, we tested several approaches to ionise and deposit nanoparticles from dispersions or suspensions containing the nanoparticles directly.

Both SDS and SoCit can be used to stabilise nanoparticles dispersed in liquids. In SDS, carbon nanotubes (CNT) were suspended, while SoCit is the main stabilizing agent in commercially available colloidal gold dispersions. These dispersions were used with the same orthogonal, pneumatic electrospray ionization source used in the cluster experiments. In both cases significant ion current could be generated. The mass spectra obtained from these ion beam, however, were identical to those of the solution of SDS and SoCit respectively.

After deposition, nanoparticles were not found on the surface. Instead only structures were observed identical to those grown from ion beams of pure SDS and SoCit solutions. Since, the islands grown from SoCit cluster can easily be mistaken for colloidal gold in shape and size[50], electron microscopy imaging and thorough growth experiments were performed, confirming the presence of only SoCit on the surface, suggesting that earlier interpretation of such structures as gold nanoparticle might be incorrect. We thus conclude that the in the ionization process only salt clusters are generated, while the nanoparticles remain neutral or extremely lowly charged.

Assuming competitive ionisation, the relatively high number concentration of stabilising agent as compared to the nanoparticles suggests that the yield would be very low. As a consequence, we tested whether the ionization of dispersed nanoparticles is enhanced when solutions without stabilizing agents like SDS or SoCit are used. For that purpose purified solutions of cadmium sulfite (CdS) nanorods (20-30 nm diameter) and vanadium oxide $\left(\mathrm{V}_{2} \mathrm{O}_{5}\right)$ nanowires (5-10 nm diameter) were electrosprayed and the ion current deposited without further mass selection, because no resolved signal was detected in the MS other than a wide region of intensity around $2000-5000 \mathrm{Th}$.

After deposition on the surface individual, globular nanoparticles of significant height were observed (Fig. 6a,b) and their chemical identity confirmed by TOF-SIMS. While the deposited worked reproducibly, none of particles on the surface has the shape of the original objects in the solution. Obviously, only fragments of the particle were ionized or transmitted, likely the consequence of fragmentation of the nanowires upon ionisation or due to the mechanical stress during the vacuum transfer, where sonic speed, high velocity gradients and shock systems can occur in the gas flow[51, 52].

Further, we attempted the deposition of carbon nanotubes, because we expect a better mechanical rigidity and hence intact ionisation and transmisstion. CNTs were dispersed in ethanol and toluol using intense ultrasonication. The resulting dispersions are stable up to an hour, so that ESI can be attempted. In fact for some experiments short nanotube fragment of up to $1 \mu \mathrm{m}$ length could be found on a $\mathrm{SiO}_{x}$ surface (Fig. 6). However, these experiments lack in reproducibility and control. Notably, the low conductivity of the solution led to visibly unstable sprays, which are represented by low and strongly fluctuating ion currents.

While the deposition of nanoparticles from solution is not reliably reproducible, it is clear that each, ionisation, transmission, and deposition, work. The lack of a fast turnover analysis, that is mass spectrometry, however, impedes the optimisation of the process, in which especially the ionisation relies on empirical adjustments to find solution- and spray conditions und which nanoparticle gas phase ions are generated. 
(a)

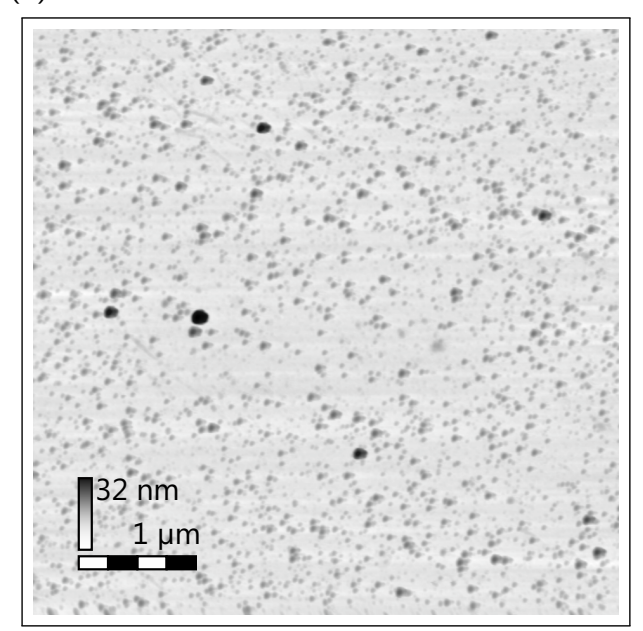

(c)

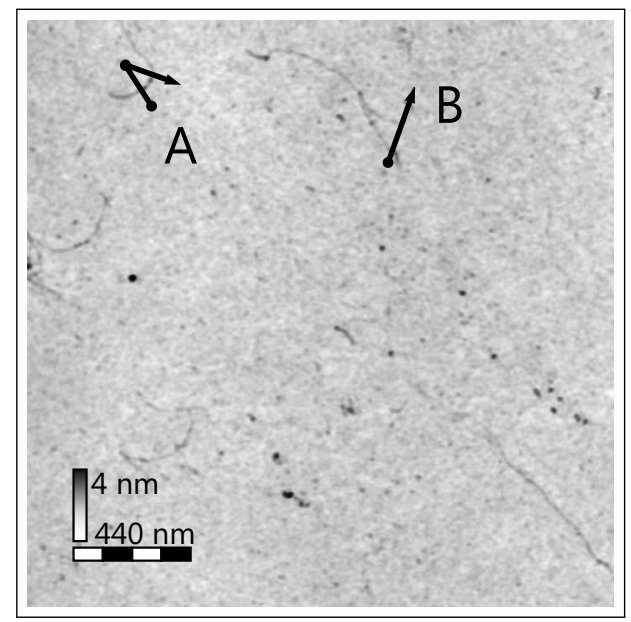

(b)

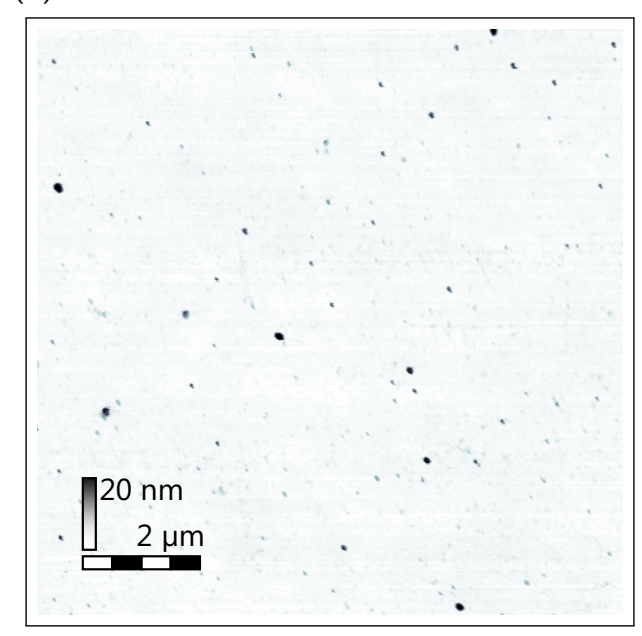

(d)
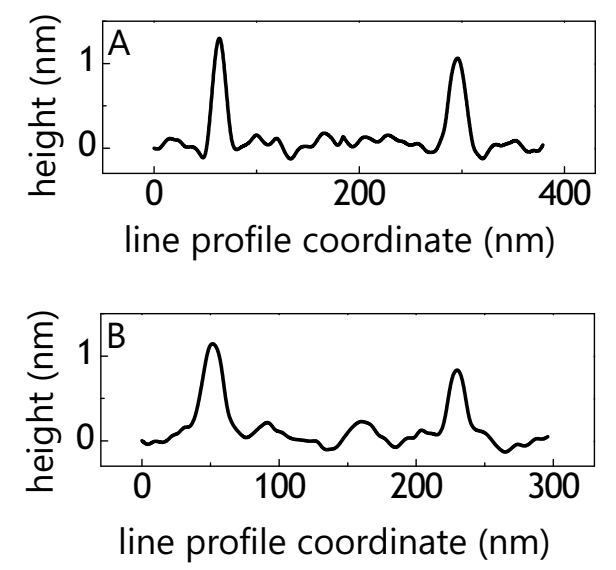

Figure 6: Deposition of Nanoparticles from dispersions. AFM topography of a $\mathrm{SiO}_{x}$ surface after deposition of nanoparticle ion beams from (a) $\mathrm{CdS}$ nanorod dispersion, (b) $\mathrm{V}_{2} \mathrm{O}_{5}$ nanowire dispersion. The original nano objects were not found, but smaller fragments. AFM topography (c) and line profile (d) of carbon nanotubes deposited on a $\mathrm{SiO}_{x}$ surface.

\section{Conclusions}

This study demonstrated that, in principle, nanoparticles or large clusters, are compatible with ES-IBD: they can be ionised from suspension or generated from a solution containing constituents, they can be transmitted and manipulated by ion optics, and finally deposited. While the structures formed on the surface allowed us to understand the properties of the beam better and transport a large amount of material with relatively low ion current, controlling the formation of structures on the surface will be essential for applications in thin film growth and nanostructure fabrication. This can be achieved by tuning the molecule-substrate interaction, which can be effectively done by controlling the substrate temperature[34,53] as well as the collision energy $[53,54]$.

In our ion beams we found clusters of several hundred molecules, which have a diameter in the range of several nanometers and thus truly represents small nanoparticles. The SDS or SoCit cluster ion beam can thus be understood as an example of a nanoparticle ion beam, which demonstrates that ES-IBD processing is also applicable to this material class. Moreover, it represents a controllable route for the fabrication of nanoparticles in the spray, provided that the aggregates formed are stable after deposition on the surface. 
Our study further shows that the intuitive evaluation of the material flux of an cluster ion beam from its mass spectrum can easily underestimate a significant contribution from large clusters of low intensity at high $m / z$ ratios. Therefore, in deposition applications, cluster formation provides the possibility of a high flux deposition as compared to pure molecular beams. Especially space charge effects, often a limiting factor of ion transmission, scale with increasing electrical current. [51, 52, 55] Hence, at the space charge limit a cluster beam carries a multiple of the material of a molecular ion beam and can be used for the fast growth of thin films or three dimensional islands as demonstrated here.

When working with large cluster ion beams one has to be aware of the appearance of nanoparticles in mass spectrometry. The mass spectra of the organic salts discussed above represent the transition from defined cluster to nanometer-sized, less defined particles. Their variation in size and charge state will lead to a distribution of low intensity peaks in the high $\mathrm{m} / \mathrm{z}$ range, which might not be resolved even by advanced mass spectrometers. While the size and charge state distribution of clusters can be manipulated in the mass spectrometer, the handling of dispersed nanoparticles was not controllable in this way. This indicates that alternative characterization methods for gas phase nanoparticles in vacuum are needed, that allow a controlled deposition of large nanoparticles on surfaces in vacuum and thus their integration in vacuum processes. Highly sensitive oscillators, nanoparticle traps or single particle mass spectrometry present viable alternatives to be integrated in future devices. [14, 41, 56-60]

Further attempts with stabilisation agent-free dispersions of CNT in ethanol and $\mathrm{V}_{2} \mathrm{O}_{5}$ and $\mathrm{CdS}$ nanowires in water-ethanol solutions were more successful, in the sense that structures were found which could be attributed to the nanoparticles - yet not reliably reproducible. Since we were able to show that in principle nanoparticles can be transmitted and deposited in ES-IBD, we conclude that the crucial step is the controlled ionization of the nanoparticles. For this, not only the chemical characterization by mass spectrometry is lacking, but also a better insight in the ionization and transport within the ion source is required. The success of native mass spectrometry $[61,62]$ and recent successful work generating nanoparticle and cluster materials in ESI and for deposition applications[38, 63-65] points towards the possibility of optimising nanoparticle ionisation, once a fast-turnaround chemical analysis (like mass spectrometry) is available.

In order to make nanoparticle deposition work reliably, finally the surface chemistry of the nanoparticle ionization in ESI must be better understood along with the transport of ions in the electrospray ion source. In particular, the large size of nanoparticle makes the interaction of the ion with the gas flow an important parameter, that is typically uncontrolled in many ion sources. Finally the possibility of in situ chemical characterization of the nanoparticle ion beams would be of great benefit to a nanoparticle ion beam deposition method.

\section{Methods}

For mass spectrometry and deposition we used our ES-IBD source.[50, 66] Mass spectra were recorded using a linear time-of-flight instrument with a discrete dynode detector (ETP 14882) and in single pulse counting mode which ensures even sensitivity over a wide mass range. Before the measurements we made sure that the mass spectra were recorded without saturating the detector. SDS (Sigma-Aldrich, 436143) and SoCit (Sigma-Aldrich, 71635) were dissolved in water and water/methanol mixtures. From these solutions, gas phase ions are created by pneumatically assisted electrospray ionization at a flow rate of $16.7 \mu \mathrm{l} / \mathrm{min}$ using a heated ESI interface at $150-180^{\circ} \mathrm{C}$. The ions are transferred into vacuum and 
guided through four differentially pumped stages to reach a pressure below $10^{-8}$ mbar at the time-of-flight mass spectrometer and sample position. On the samples ion currents of $20-250$ pA were reached and decelerated to a kinetic energy of $2-5 \mathrm{eV}$ per charge before deposition. The beam focus spot was chosen very wide to obtain a homogenous coverage on the sample, behind the fixed diameter deposition aperture which crops only the central region of the gaussian beam profile. The coverage is monitored via a current measurement at the sample position using electrometers (Keithley 617). The HOPG substrates were cleaved at ambient conditions before transferring into vacuum for deposition. AFM was acquired at ambient conditions in tapping mode directly after deposition. The images were analyzed with the WSXM software.[67]

\section{supplementary material}

Experimental method details and further information concerning the calculation, fitting routine and nanoparticle deposition attempts are available in the supporting information.

(1) Alivisatos, A. Endeavour 1997, 21, 56.

(2) Talapin, D. V.; Lee, J.; Kovalenko, M. V.; Shevchenko, E. V. Chem. Rev. 2010, 110, 389-458.

(3) Fenton, J. L.; Steimle, B. C.; Schaak, R. E. Science 2018, 360, 513-517.

(4) Milani, P.; S., I., Cluster Beam Synthesis and Nanostructured Materials; Springer: 1999.

(5) Bromann, K.; Felix, C.; Brune, H.; Harbich, W.; Monot, R.; Buttet, J.; Kern, K. Science 1996, 274, 26, $956-958$.

(6) Bromann, K.; Brune, H.; Felix, C.; Harbich, W.; Monot, R.; Buttet, J.; Kern, K. Surface Science 1997, 377, 16, 10511055.

(7) Böttcher, A.; Weis, P.; Bihlmeier, A.; Kappes, M. M. Phys. Chem. Chem. Phys. 2004, 6, 5213 ?5217.

(8) Cheng, H. P.; Landman, U. Science 1993, 260, 38, 1304-1307.

(9) Röttgen, M.; Judai, K.; Antonietti, J.-M.; Rauschenbach, S.; Kern, K.; Heiz U., R. S. Rev. Sci. Instr. $2006,77,013302$.

(10) Benjamin, D. R.; Robinson, C. V.; Hendrick, J. P.; Hartl, F. U.; Dobson, C. M. Proc. Natl. Acad. Sci. USA 1998, 95, 7391-7395.

(11) Fuerstenau, S. D.; Benner, W. H.; Thomas, J. J.; Brugidou, C.; Bothner, B.; Siuzdak, G. Angew. Chem., Int. Ed. 2001, 40, 982-982.

(12) Snijder, J.; Rose, R. J.; Veesler, D.; Johnson, J. E.; Heck, A. J. R. Angew. Chem., Int. Ed., 2013, 52, $4020-4023$.

(13) Howder, C. R.; Bell, D. M.; Anderson, S. L. Review of Scientific Instruments 2014, 85, 014104.

(14) Esser, T. K.; Hoffmann, B.; Anderson, S. L.; Asmis, K. R. Review of Scientific Instruments 2019, 90, 125110.

(15) Kane, B. E. Phys. Rev. B 2010, 82, 115441.

(16) Gamero-Castano, M.; de la Mora, J. F. Analytica Chimica Acta 2000, 406, 61, 67-91.

(17) Lenggoro, I. W.; Xia, B.; Okuyama, K.; de la Mora, J. F. Langmuir 2002, 18, 4584-4591.

(18) Meng, C.; Fenn, J. B. Organic Mass Spectrometry 1991, 26.

(19) Zhang, D. X.; Cooks, R. G. International Journal of Mass Spectrometry 2000, 196, 667-684.

(20) Zhou, S. L.; Hamburger, M. Rapid Communications in Mass Spectrometry 1996, 10, 19, 797-800.

(21) Juraschek, R.; Dulcks, T.; Karas, M. Journal of the American Society for Mass Spectrometry 1999, 10, 25, 300-308.

(22) Sharon, M.; Ilag, L. L.; Robinson, C. V. J. Am. Chem. Soc. 2007, 129, 8740-8746.

(23) Rauschenbach, S.; Rinke, G.; Malinowski, N.; Weitz, R. T.; Dinnebier, R.; Thontasen, N.; Deng, Z.; Lutz, T.; de Almeida Rollo, P. M.; Costantini, G.; Harnau, L.; Klaus Kern, R. S. Adv. Mater. 2012, 24, 2761-2767.

(24) Vats, N.; Wang, Y.; Sen, S.; Szilagyi, S.; Ochner, H.; Abb, S.; Burghard, M.; Sigle, W.; Kern, K.; van Aken, P. A.; Rauschenbach, S. ACS Nano 2020, 14, 4626-4635.

(25) Johnson, G. E.; Wang, C.; Priest, T.; Laskin, J. Anal. Chem. 2011, 83, 8069-8072.

(26) Swarbrick, J. C.; Ben Taylor, J.; O'Shea, J. N. Appl. Surf. Sci. 2006, 252, 29, 5622-5626.

(27) O'Shea, J. N.; Taylor, J. B.; Swarbrick, J. C.; Magnano, G.; Mayor, L. C.; Schulte, K. Nanotechnology 2007, 18, O'Shea, James N. Taylor, John B. Swarbrick, Janine C. Magnano, Graziano Mayor, Louise C. Schulte, Karina 17, 4.

(28) Therrien, J.; Dindar, A.; Smith, D. Microscopy Research and Technique 2007, 70, 530-533.

(29) Tanaka, H.; Kawai, T. Nature Nanotechnology 2009, 4, 518-522.

(30) Johnson, G. E.; Hu, Q.; Laskin, J. Annu. Rev. Anal. Chem. 2011, 4, 83-104. 
(31) Rauschenbach, S.; Ternes, M.; Harnau, L.; Kern, K. Annu. Rev. Anal. Chem. 2016, 9, 16.1-16.26.

(32) Vats, N.; Rauschenbach, S.; Sigle, W.; Sen, S.; Abb, S.; Portz, A.; Durr, M.; Burghard, M.; van Aken, P.; Kern, K. Nanoscale 2018, 10, 4952-4961.

(33) Abb, S.; Tarrat, N.; Cortés, J.; Andriyevsky, B.; Harnau, L.; Schön, J. C.; Rauschenbach, S.; Kern, K. RSC Adv 2019, 9, 35813-35819.

(34) Wu, X.; Delbianco, M.; Anggara, K.; Michnowicz, T.; Pardo-Vargas, A.; Bharate, P.; Sen, S.; Pristl, M.; Rauschenbach, S.; Schlickum, U.; Abb, S.; Seeberger, P.; Kern, K. Nature 2020, 582, 375-378.

(35) Thontasen, N.; Levita, G.; Malinowski, N.; Deng, Z.; Rauschenbach, S.; Kern Klaus, R. S. J. Phys. Chem. C 2010, 114, 17768-17772.

(36) Kley, C. S.; Dette, C.; Rinke, G.; Patrick, C. E.; Cechal, J.; Jung, S. J.; Baur, M.; Duerr, M.; Rauschenbach, S.; Giustino, F.; Stepanow, S.; Kern, K. Nano Lett. 2014, 14, 563-569.

(37) Dubey, G.; Urcuyo, R.; Abb, S.; Rinke, G.; Burghard, M.; Rauschenbach, S.; Klaus Kern, R. S. J Am Chem Soc 2014, 136, 13482-13485.

(38) Prabhakaran, V.; Mehdi, B. L.; Ditto, J. J.; Engelhard, M. H.; Wang, B.; Don, K.; Gunaratne, D.; Johnson, D. C.; Browning, N. D.; Johnson, G. E.; Laskin, J. Nat Commun 2016, 7, 11399.

(39) Chorev, D. S.; Tang, H.; Rouse, S. L.; Bolla, J. R.; von Kügelgen, A.; Baker, L. A.; Wu, D.; Gault, J.; Grünewald, K.; Bharat, T. A., et al. Nat. Protoc. 2020, 15, 1690-1706.

(40) Gault, J.; Donlan, J. A.; Liko, I.; Hopper, J. T.; Gupta, K.; Housden, N. G.; Struwe, W. B.; Marty, M. T.; Mize, T.; Bechara, C., et al. Nat Methods 2016, 13, 333-336.

(41) Keifer, D. Z.; Jarrold, M. F. Mass Spectrometry Reviews 2017, 36, 715-733.

(42) Cooks, R. G.; Rockwood, A. L. Rapid Commun. Mass Spectrom. 1991, 5, 93.

(43) Williams, R. J.; Phillips, J. N.; Mysels, K. J. Trans. Faraday Soc. 1955, 51, 728-737.

(44) Bales, B. L.; Messina, L.; Vidal, A.; Peric, M.; Nascimento, O. R. J. Phys. Chem. B 1998, 102, 10347-10358.

(45) Wang, Y.; Larsson, D. S. D.; van der Spoel, D. Biochemistry 2009, 48, 1006-1015.

(46) van der Spoel, D.; Marklund, E. G.; Larsson, D. S. D.; Caleman, C. Macromol. Biosci. 2011, 11, 50-59.

(47) Jaynes, E. T., Probability theory; Cambridge University Press: 2006.

(48) Martin, T. P. Phys. Rep. 1996, 273, 199-241.

(49) Smith, L. A.; Duncan, A.; Thomson, G. B.; Roberts, K. J.; Machin, D.; McLeod, G. Journal of Crystal Growth 2004, 263, 480.

(50) Rauschenbach, S.; Stadler, F. L.; Lunedei, E.; Malinowski, N.; Koltsov, S.; Costantini, G.; Kern K., R. S. Small 2006, 2, 540-547.

(51) Bernier, L.; Pinfold, H.; Pauly, M.; Rauschenbach, S.; Reiss, J. J. Am. Soc. Mass. Spectrom. 2018, $29,761-773$.

(52) Pauly, M.; Sroka, M.; Reiss, J.; Rinke, G.; Albarghash, A.; Vogelgesang, R.; Hahne, H.; Kuster, B.; Sesterhenn, J.; Kern, K.; Rauschenbach S., R. S. Analyst 2014, 139, 1856-1867.

(53) Anggara, K.; Zhu, Y.; Delbianco, M.; Rauschenbach, S.; Abb, S.; Seeberger, P. H.; Kern, K. J Am Chem Soc 2020 , 142, 21420-21427.

(54) Krumbein, L.; Anggara, K.; Stella, M.; Michnowicz, T.; Ochner, H.; Abb, S.; Rinke, G.; Portz, A.; Dürr, M.; Schlickum, U.; Baldwin, A.; Floris, A.; Kern, K.; Rauschenbach, S. Phys Rev Lett 2021, 126, 056001.

(55) Bernier, L.; Taesch, M.; Rauschenbach, S.; Reiss, J. Int. J. Mass Spectrom. 2020, 447, 116239.

(56) Schlemmer, S.; Wellert, S.; Windisch, F.; Grimm, M.; S., B.; D., G. Appl. Phys. A 2004, 78, 629-636.

(57) Grimm, M.; Langer, B.; Schlemmer, S.; Lischke, T.; Widdra, W.; Gerlich, D.; Becker, U.; R?hl, E. AIP Conf. Proc. 2004, 705, 1062-1065.

(58) Hanay, M. S.; Kelber, S.; Naik, A. K.; Chi, D.; Hentz, S.; Bullard, E. C.; Colinet, E.; Duraffourg, L.; Roukes, M. L. Nature Nanotechnology 2012, 7, 602-608.

(59) Jensen, K.; Kim, K.; Zettl, A. Nature Nanotechnology 2008, 3, 533-537.

(60) Wörner, T. P.; Snijder, J.; Bennett, A.; Agbandje-McKenna, M.; Makarov, A. A.; Heck, A. J. Nature Methods 2020, 17, 395-398.

(61) Heck, A. J. R. Nat. Methods 2008, 5, 927.

(62) Benesch, J. L. P.; Ruotolo, B. T.; Simmons, D. A.; Robinson, C. V. Chem. Rev. 2007, 107, 3544-3567.

(63) Marsh, B. M.; lyer, K.; Cooks, R. G. J Am Soc Mass Spectrom 2019, 30, 2022-2030.

(64) Li, A.; Luo, Q.; Park, S.-J.; Cooks, R. G. Angew Chem Int Ed 2014, 53, 3147-3150.

(65) Li, A.; Baird, Z.; Bag, S.; Sarkar, D.; Prabhath, A.; Pradeep, T.; Cooks, R. G. Angew Chem Int Ed 2014, n/a-n/a. 
(66) Rauschenbach, S.; Vogelgesang, R.; Malinowski, N.; Gerlach, J. W.; Benyoucef, M.; Costantini, G.; Deng, Z.; Thontasen, N.; Kern Klaus, R. S. ACS Nano 2009, 3, 2901-2910.

(67) Horcas, I. Rev Sci Instrum 2007, 78, 013705. 

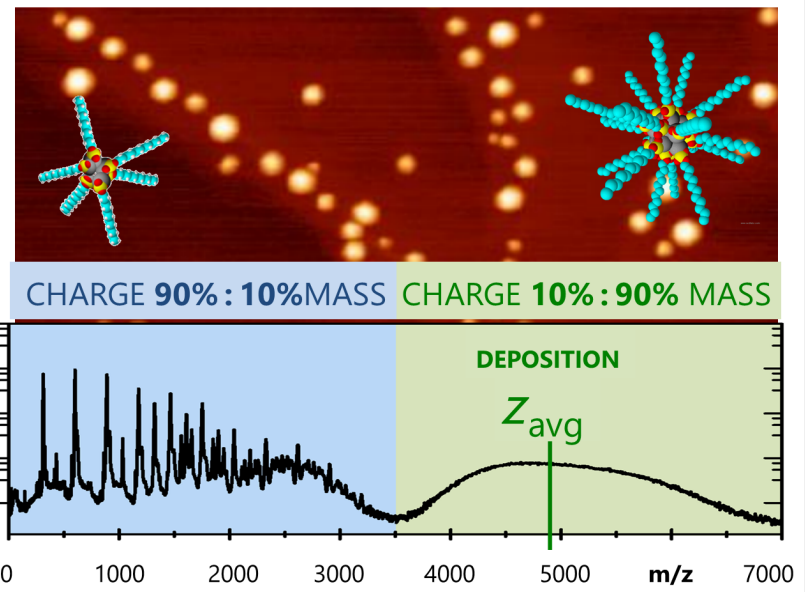

Table of Contents Figure. In molecular cluster ion beams, small clusters carry the major amount of charge, while large clusters carry the major amount of material. 